\title{
Predictive and Prognostic Significance of p27, Akt, PTEN and PI3K Expression in HER2-Positive Metastatic Breast Cancer
}

\author{
Kerem Okutur ${ }^{1 *}$, Nuray Bassulu², Levent Dalar ${ }^{3}, K_{\text {Kubra Aydin }}^{4}$, Mustafa \\ Bozkurt $^{1}$, Kezban Nur Pilanci ${ }^{5}$, Gulen Bulbul Dogusoy ${ }^{2}$, Coskun Tecimer ${ }^{5}$, Nil \\ Molinas Mandel ${ }^{6}$, Gokhan Demir ${ }^{1}$
}

\begin{abstract}
Background: The phosphatidylinositol 3'-kinase/Akt (PI3K/Akt) pathway is a key regulator for HER2overexpressing breast cancer, but data about whether activation of PI3K/Akt is associated with poor prognosis and resistance to trastuzumab therapy is controversial. In this study we investigated predictive and prognostic significance of expression of p27, Akt, PTEN and PI3K, which are components of the PI3K/Akt signaling pathway, in HER2-positive metastatic breast cancer (MBC), retrospectively. Materials and Methods: Fifty-four HER2positive MBC patients who had received first-line trastuzumab-based therapy were recruited for the study group. All of the patient's breast tissue samples were examined for p27 and Akt expression. In addition, twenty-five patients with sufficient amount of tumor tissue were also examined for PTEN and PI3K expression. p27, Akt, PTEN and PI3K were evaluated by immunohistochemistry and their relationship with patient demographic features, tumor characteristics, response to trastuzumab-based treatment and survival outcomes were analyzed. Results: p27, Akt, PTEN and PI3K were positive in $25.9 \%, 70.4 \%, 24 \%$ and $96 \%$ of the cases, respectively. Nomne were significantly associated with response to trastuzumab and time to progression (TTP). A trend toward statistical significance for longer overall survival (OS) was found for PTEN-positive patients $(p=0.058)$; there was no significant relationship between the other immunohistochemical variables and $O S$. When we analyzed groups regarding co-expression, the PTEN-negative/Akt-negative group had a significantly lower objective response rate (ORR) $(20 \%$ vs $80 \%, \mathrm{p}=0.023)$ and the PTEN-negative/p27-negative and PTEN-negative/Akt-negative groups had significantly lower median OS compared to other patients (26.4 months $v$ s 76.1 months, $p=0.005$ and 25.6 months vs 52.0 months, p=0.007, respectively). Conclusions: p27, Akt, PTEN and PI3K expression is not statistically significantly associated with ORR, TTP and OS, individually. However, the combined evaluation of p27, Akt and PTEN could be helpful to predict the response to trastuzumab-based therapy and prognosis in HER2-positive MBC.
\end{abstract}

Keywords: Breast cancer - HER2, p27 - Akt - PTEN - trastuzumab - response to therapy

Asian Pac J Cancer Prev, 16 (7), 2645-2651

\section{Introduction}

HER2 is a transmembrane glycoprotein encoded by the HER2/neu (c-erbB-2) protooncogen located on the long arm of the 17 th chromosome, with a molecular weight of $185 \mathrm{kD}$ and consisting of 1255 amino acids (Chen et al., 2014). HER2 gene plays a role in controlling cellular growth, division and repair. HER2 amplification and/or protein overexpression is found in $20-30 \%$ of patients with newly diagnosed breast cancer and is associated with more aggressive clinical course and shorter survival rates when compared with patients with normal HER2 levels (Liu et al., 2012). Amplification and overexpression lead continuous stimulation of receptor tyrosine kinase signal pathways known as Ras/Raf/MEK/MAPK and phosphatidylinositol 3'-kinase/Akt (PI3K/Akt). This in turn triggers a cascade of downstream cellular functions such as uncontrolled cell proliferation, invasiveness, resistance to apoptosis and angiogenesis (Ross et al., 2009).

Identification of HER2 as an oncogene in breast cancer has enabled the development of targeted therapies. Trastuzumab is a humanized monoclonal antibody which acts by binding to the extracellular domain of HER2. Since it was shown that HER2 provides clinical response when used alone and prolongs survival when combined with chemotherapy in metastatic patients with HER2 overexpression, it has become a "sine qua non" therapeutic

${ }^{1}$ Department of Medical Oncology, Acibadem University School of Medicine, ${ }^{2}$ Department of Pathology, ${ }^{3}$ Department of Chest Disease, Istanbul Bilim University School of Medicine, Department of Medical Oncology, ${ }^{6}$ Koc University School of Medicine , Istanbul, ${ }^{4}$ Ankara Oncology Training and Research Hospital, ${ }^{5}$ Istanbul Bilim University School of Medicine, Ankara, Turkey *For correspondence: keremokutur@gmail.com 
agent for this patient group (Spector and Blackwell, 2009). Currently, use of trastuzumab has become a standard therapy in the adjuvant therapy of early stage HER2positive breast cancer combined with chemotherapy and in advanced stage disease combined with pertuzumab as part of dual blockage (Prat and Baselga, 2013).

Despite all these favorable effects, approximately 40-50\% of HER2-positive breast cancer patients do not respond to trastuzumab-containing therapies. In addition, although most of the patients respond to trastuzumab-based therapies initially, the disease usually progresses within the first year. On the other hand, metastatic disease occurs in approximately $15 \%$ of patients receiving adjuvant trastuzumab (Nahta and Esteva, 2006). Understanding the mechanisms underlying this "trastuzumab resistance" is important regarding identification of patients that would benefit most from therapy.

Trastuzumab disrupts signal transmissions in the PI3K/Akt and MAPK cascade pathways by binding to HER2. Molecules located in these pathways are thought to be associated with unresponsiveness to trastuzumab. Studies involving phosphatase and tensin homologue (PTEN) which is a tumor suppressor gene and the most important regulator of PI3K/Akt pathway were followed by other studies involving PI3K, p95, p27, Akt, IGF-1R, MUC-4 and c-met, but results of the studies are conflicting (Fiszman and Jasnis, 2011).

In this study, we retrospectively analyzed the association of p27, Akt, PTEN and PI3K expressions with response to treatment and survival in HER2-positive metastatic breast cancer (MBC) patients receiving firstline trastuzumab-based therapy.

\section{Materials and Methods}

Histologically diagnosed HER2-positive MBC patients who received first-line trastuzumab-based therapy between February 2002 and October 2011 were included in the study. Association of p27 and Akt expression detected with immunohistochemistry (IHC) in the breast tissue blocks of the patients with disease characteristics, response to therapy and prognosis were investigated. Hematoxylene eosin (HE) sections were taken from the paraffin breast tissue blocks. Blocks containing components of invasive carcinoma were selected and IHC was applied to these sections In addition p27 and Akt expressions were studied PTEN and PI3K expressions were also studied in patients with still adequate tumor tissue. The study was approved by the local ethics committee.

\section{Immunohistochemistry procedure and scoring}

4- $\mu \mathrm{m}$-thick sections were taken from parafinembedded tissue blocks fixated with $10 \%$ formaldehyde. HE staining was applied to one section from each block. Immunohistochemical staining was carried out with the streptavidin-enhanced immunoperoxidase technique with Leica Bond Max automatic staining device using PI3K (PAB3212, Abnova) diluted at 1/50, p27 (1B4, Novacastra, NCL-P27) diluted at 1/20, PTEN (28H6,Gene Tex, GTX73862) diluted at 1/60 and Akt (NCL-L-AktPhos Clone LP18) primary antibodies diluted at 1/50.
Following deparaffinization, citrate buffer with a $\mathrm{pH}$ of 6.0 was applied to p27, PTEN and Akt antibodies, and EDTA with a pH of 8.0 to the PI3K antibody by the antigen retrieval protocol. As chromogenic substrates, 3-amino-9-ethylcarbazole (AEC) was used for p27,PTEN and Akt, and diaminobenzidine (DAB) for PI3K. Sections containing p27, PTEN and Akt were closed with aques mounting medium and sections containing PI3K with xylene consul-mount.

All HE and IHC stained sections were examined by two pathologists who were blinded to the histologic characteristics of the tumors. Nuclear and/or cytoplasmic staining was obtained with PTEN, nuclear with p27, cytoplasmic with PI3K, nuclear and/or cytoplasmic with Akt. As controls, normal prostate tissue was used for PTEN, normal tonsil tissue for p27, mammary tissue containing carcinoma for PI3K and normal skin tissue for

Table 1. Clinical and Pathologic Characteristics of 54 HER2-Positive MBC Treating with TrastuzumabBased Therapies

\begin{tabular}{|c|c|c|}
\hline & $\mathrm{N}$ & $(\%)$ \\
\hline Median age (range) & $-80)$ & \\
\hline \multicolumn{3}{|l|}{ ECOG performance status } \\
\hline 0 & 23 & 42.6 \\
\hline 1 & 26 & 48.1 \\
\hline 2 & 5 & 9.3 \\
\hline \multicolumn{3}{|l|}{ Menopausal status } \\
\hline Premenopausal & 13 & 24.1 \\
\hline Postmenopausal & 41 & 75.9 \\
\hline \multicolumn{3}{|l|}{ Primary tumor } \\
\hline Not resected & 9 & 16.7 \\
\hline Resected & 45 & 83.3 \\
\hline \multicolumn{3}{|l|}{ Histology } \\
\hline Ductal & 50 & 92.6 \\
\hline Ductal+lobular & 3 & 5.6 \\
\hline Lobular & 1 & 1.9 \\
\hline \multicolumn{3}{|l|}{ Grading } \\
\hline Grade 2 & 30 & 55.6 \\
\hline Grade 3 & 24 & 44.4 \\
\hline \multicolumn{3}{|l|}{ Hormone receptor status } \\
\hline Negative & 17 & 31.5 \\
\hline Positive & 37 & 68.5 \\
\hline \multicolumn{3}{|l|}{ ER/PR status } \\
\hline $\mathrm{ER}+/ \mathrm{PR}+$ & 27 & 50.0 \\
\hline $\mathrm{ER}+/ \mathrm{PR}-$ & 10 & 18.5 \\
\hline ER-/PR- & 17 & 31.5 \\
\hline \multicolumn{3}{|l|}{ Distant metastatic sites } \\
\hline Bone-soft tissue & 24 & 44.4 \\
\hline Visceral & 30 & 55.6 \\
\hline \multicolumn{3}{|l|}{ Number of metastatic sites } \\
\hline 1 & 29 & 53.7 \\
\hline 2 & 22 & 40.7 \\
\hline$\geq 3$ & 3 & 5.6 \\
\hline Brain metastasis at the initial presentation & 4 & 7.4 \\
\hline Adjuvant chemotherapy & 28 & 51.9 \\
\hline Adjuvant trastuzumab & 15 & 27.8 \\
\hline \multicolumn{3}{|c|}{ First line chemotherapy regimen with trastuzumab } \\
\hline Taxanes & 39 & 72.2 \\
\hline Vinorelbine & 4 & 7.4 \\
\hline Capecitabine & 3 & 5.6 \\
\hline Hormonal therapies & 8 & 14.8 \\
\hline Trastuzumab after progression & 36 & 66.7 \\
\hline Lapatinib after progression & 17 & 31.5 \\
\hline
\end{tabular}


Predictive and Prognostic Significance of p27, Akt, PTEN and PI3K Expression in HER2-Positive Metastatic Breast Cancer

Akt. Scoring was carried out because staining intensities were different.

PTEN was scored semiquantitatively using the immunoreactive score (IRS) as described by Nagata et al. (2004). Result obtained by multiplying the percentage of positively stained cells with staining intensity was recorded as IRS. Staining intensity was graded as 0 (negative), 1 (poor), 2 (moderate), 3 (strong); percentage of positive staining was scored $0(<5 \%), 1(5 \%-25 \%)$, $2(26 \%-50 \%), 3(51 \%-75 \%), 4(>75 \%)$. Based on the values obtained, IRS of 0-3 was recorded as 0 (expression absent), IRS of 4-6 as 1+ (low expression), IRS of 7-9 as $2+$ (intermediate expression), IRS of $10-12$ as $3+$ (high expression). If IRS was $<7$, PTEN was considered negative; if $\geq 7$, PTEN was considered positive. If the proportion of cells exhibiting positive staining for $\mathrm{p} 27$ and $\mathrm{PI} 3 \mathrm{~K}$ was $\geq 50 \%$, it was considered positive staining, and if $<50 \%$, it was negative staining (Spataro et al., 2003; Fabi et al., 2010). Since there is no validated scoring system for Akt expression, if the proportion of cells exhibiting positive staining was $\geq 50 \%$, it was considered positive staining, and if $<50 \%$, it was negative staining.

\section{Evaluation of the response}

Treatment response was evaluated in all patients with computerized tomography every 8-12 weeks after the initiation of therapy. The Response Evaluation Criteria in Solid Tumours (RECIST) version 1.1 was used for evaluating the status of the response. Objective response rate (ORR) was defined as the proportion of complete response (CR) and partial response (PR) in all patients. Disease control rate (DCR) was classified as the percentage of the patients with objective response and stable disease (SD). Time to progression (TTP) was defined as the time from initiation of trastuzumab-based therapy to the date of detection of disease progression and overall survival (OS) as time from onset of trastuzumab-based therapy to death due to any cause or last day of the follow-up.

\section{Statistical analysis}

Statistical Package for the Social Sciences (SPSS) version 16.0 program was used for statistical analysis. In the evaluation of study data, in addition to descriptive statistical methods (mean, standard deviation, median, frequency, rate, minimum, maximum), Student-t test was used for comparing variables showing normal distribution and Mann Whitney U test for comparing variables not showing normal distribution within qualitative data. Pearson's Chi-Square test, Fisher's Exact Test and Yates Continuity Correction Test were used for comparing qualitative data. Kaplan Meier survival analysis and log-rank were used for evaluating survival. Statistical significance was evaluated at the level of $\mathrm{p}<0.05$.

\section{Results}

\section{Patient and tumor characteristics}

Clinical and pathologic characteristics of 54 patients included in the study are shown in Table 1. Median age was 57 (28-80) years. Performance status was ECOG 0-1 in $91 \%$ of the patients in the study group. Forty-one patients
$(75.9 \%)$ were postmenopausal and hormone receptors were positive in 37 patients $(68.5 \%)$. Thirty patients $(55.6 \%)$ had visceral disease and four patients $(7.4 \%)$ had brain metastases at diagnosis. Twenty-eight patients (51.9\%) had previously received adjuvant chemotherapy and 15 patients $(27.8 \%)$ adjuvant trastuzumab. When agents used in conjunction with trastuzumab in the first-line therapy were analyzed, 46 patients $(85 \%)$ had received chemotherapy and 8 patients $(15 \%)$ hormonal therapy. The most common chemotherapeutic agent used with trastuzumab was the taxan group (paclitaxel and docetaxel). Rate of trastuzumab-based therapy use following progression was $66.7 \%(n=36)$ and of lapatinib use was $31.5 \%(\mathrm{n}=17)$.

\section{p27, Akt, PTEN and PI3K expression}

p27 ve Akt expression was studied in all of fifty-four patients. After studying p27 and Akt expression, PTEN and PI3K expression was also studied in 25 patients with sufficient tumor tissue. Immunohistochemical data of

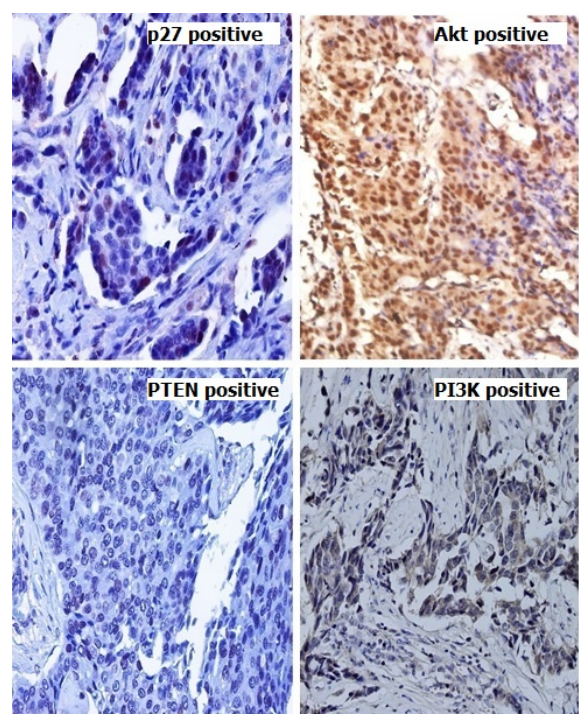

Figure 1. Immunohistochemically positive p27, Akt, PTEN ve PI3K expression

Table 2.TTPand OSAccording to Immunohistochemical Variables

\begin{tabular}{|c|c|c|c|c|}
\hline & $\begin{array}{c}\text { Median TTP } \\
\text { (months) }\end{array}$ & $\mathrm{p}$ & $\begin{array}{c}\text { Median OS } \\
\text { (months) }\end{array}$ & $\mathrm{p}$ \\
\hline \multicolumn{5}{|l|}{ P27 status } \\
\hline Negative & 11.6 & 0.485 & 42.8 & 0.992 \\
\hline Positive & 8.0 & & 52.0 & \\
\hline \multicolumn{5}{|l|}{ Akt status } \\
\hline Negative & 9.8 & 0.209 & 28.4 & 0.915 \\
\hline Positive & 10.9 & & 46.7 & \\
\hline \multicolumn{5}{|l|}{ PTEN status } \\
\hline Negative & 10.8 & 0.174 & 26.4 & 0.058 \\
\hline Positive & 21.2 & & 76.1 & \\
\hline \multicolumn{5}{|l|}{ PTEN expression } \\
\hline Absent & 10.8 & & 26.4 & \\
\hline Low & 5.4 & 0.108 & 25.6 & 0.307 \\
\hline Intermediate & 26.3 & & 76.1 & \\
\hline High expression & 21.2 & & 52.0 & \\
\hline \multicolumn{5}{|l|}{ PI3K status } \\
\hline Negative & 18.8 & 0.834 & - & 0.228 \\
\hline Positive & 11.0 & & - & \\
\hline
\end{tabular}


Table 3. ORR, TTP and OS According to Co-Expression of Immunohistochemical Variables

\begin{tabular}{|c|c|c|c|c|c|c|}
\hline & $\begin{array}{c}\text { ORR } \\
(\%)\end{array}$ & $\mathrm{p}$ & $\begin{array}{l}\text { MedianTTP } \\
\text { (months) }\end{array}$ & $\mathrm{p}$ & $\begin{array}{c}\text { Median OS } \\
\text { (months) }\end{array}$ & $\mathrm{p}$ \\
\hline $\mathrm{PTEN}+/ \mathrm{p} 27+(\mathrm{n}=3)$ & 100 & & $26 / 3$ & & 63.7 & \\
\hline PTEN+/p27- $(\mathrm{n}=2)$ & 100 & & $18 / 8$ & & 100.0 & \\
\hline PTEN-/p27+ (n=3) & 66.7 & & 6.0 & & 126.6 & \\
\hline PTEN-/p27- $(\mathrm{n}=17)$ & 58.8 & & 11.0 & & $26 / 4$ & \\
\hline PTEN-/p27 $(\mathrm{n}=17)$ vs others $(\mathrm{n}=8)$ & 58.8 vs 41.2 & 0.205 & $11.0 v s 18.8$ & 0.398 & 26.4 vs 76.1 & 0.005 \\
\hline PTEN+/Akt+ $(\mathrm{n}=3)$ & 100 & & $16 / 2$ & & 52.0 & \\
\hline PTEN+/Akt- $(\mathrm{n}=4)$ & 100 & & $18 / 8$ & & 63.7 & \\
\hline PTEN-/Akt+ $(\mathrm{n}=13)$ & 69.2 & & $12 / 2$ & & 42.8 & \\
\hline PTEN-/Akt- $(\mathrm{n}=5)$ & 20 & & $5 / 4$ & & $25 / 6$ & \\
\hline PTEN-/Akt- $(\mathrm{n}=5) v s$ others $(\mathrm{n}=20)$ & 20 vs 80 & 0.023 & $5.4 v s 13.3$ & 0.054 & 25.6 vs 52.0 & 0.007 \\
\hline
\end{tabular}
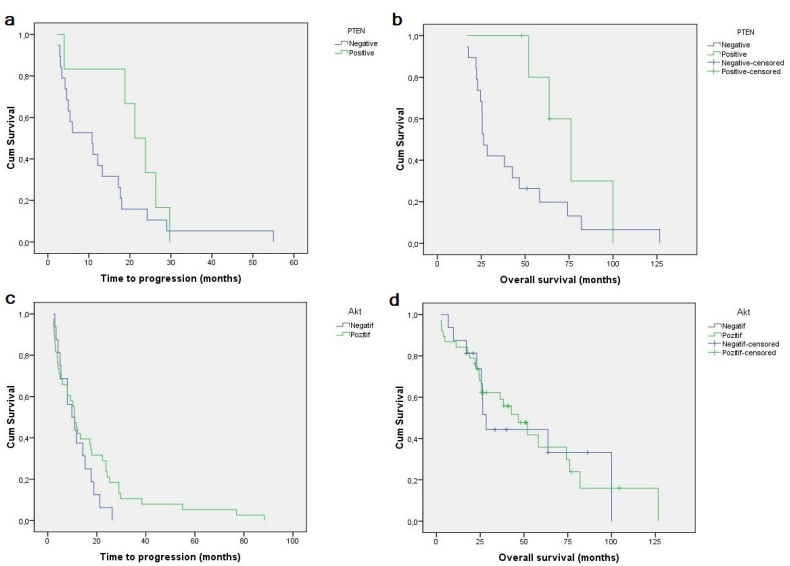

Figure 2. Kaplan-Meier Estimates of PFS and OS According to Expression of PTEN and Akt
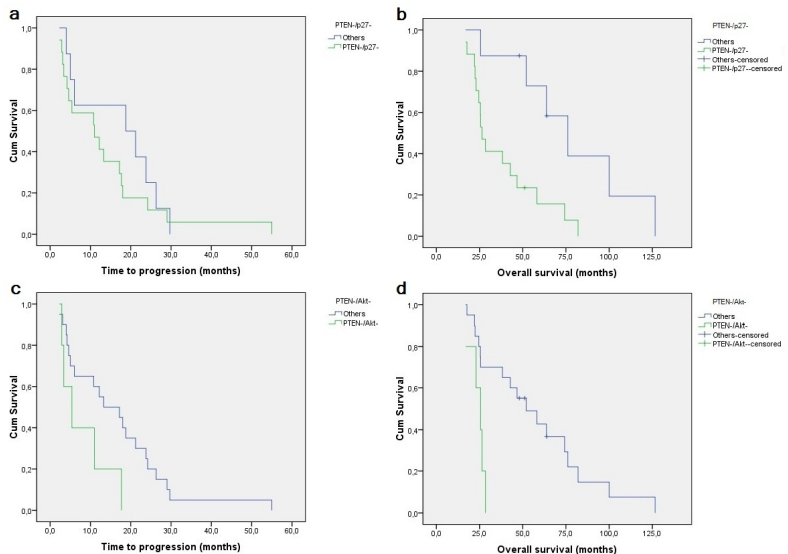

Figure 3. Kaplan-Meier Estimates of TTP and OS According to Co-Expression Groups Including PTEN-/ p27- and PTEN-/Akt-

the study group are shown in Figure 1. Fourteen patients (25.9\%) were p27 positive and 38 patients $(70.4 \%)$ Akt positive. Six patients (24\%) were PTEN positive according to the Nagata scoring system. When PTEN expression was evaluated taking into account the IRS, 6 patients (24\%) had low expression, 3 had (12\%) intermediate expression, 3 had (12\%) high expression; 13 patients $(52 \%)$ had no expression. PI3K was positive in 24 of 25 patients (96\%).

Treatment response and its relationship with clinicopathologic parameters, survival outcomes and immunohistochemical results

All of fifty-four patients were eligible for evaluating the response to trastuzumab-based therapy. ORR was
$51.9 \%$ (CR in two patients and PR in 26 patients), SD was $27.8 \%(n=15)$ and DCR was $79.6 \%(n=43)$. Eleven patients $(27.4 \%)$ had progressive disease (PD).

There were no statistically significant differences between median age, menopausal status, ECOG status, grade, status of hormone receptors, ER/PR status, sites of distant metastases, number of metastatic sites, prior adjuvant chemotherapy, adjuvant trastuzumab therapy, treatment regimens received with trastuzumab, p27 expression, Akt expression, PTEN expression and PI3K expression and response to trastuzumab-based therapy. Rate of invasive ductal carcinoma was significantly higher in the patient group with objective response compared with the non-responders ( $100 \%$ vs $86 \%, \mathrm{p}=0.047)$. Additionally, rate of initial brain metastases were significantly lower in the patient group with objective responses compared with the non-responders ( $0 \%$ vs $15.4 \%, \mathrm{p}=0.047)$.

Both TTP and OS were found to be significantly longer in patients responsive to trastuzumab-based therapy. Median TTP was 15.2 months (range 4-55 months) in responders and 5.2 months (range 5.2-88.4 months) in non-responders $(\mathrm{p}=0.005)$. Median OS was 41.6 months (range 6.8-126.6 months) in the patient group responsive to trastuzumab and, 25.1 months (range 2.5-104.4 months) in non-responders $(\mathrm{p}=0.009)$.

Survival outcomes according to clinicopathologic variables and immunhistochemical parameters

During a median follow-up of 28.5-months (range 2.5126.6 months) thirtyfour of 54 patients (63\%) died. Cause of death was disease progression in all of the patients. No significant relationship was found between TTP and OS and age $(\leq 60$ and $>60)$, menopausal status, grade, status of hormone receptors, number of metastatic sites, prior adjuvant chemotherapy, prior adjuvant trastuzumab, firs-line treatment regimens applied with trastuzumab. There was a significant association between median TTP and tumor histology and ER/PR status. The patients with ductal histology and $\mathrm{ER}+\mathrm{PR}+$ group had longer median TTP $(p=0.024$ and $p=0.044$, respectively, data not shown). Additionally, there was a significant correlation between median OS and ECOG PS, distant metastatic sites and initial brain metastasis. The group with ECOG PS 0, those with bone-soft tissue as distant metastatic site and those without initial brain metastases had longer median OS $(\mathrm{p}=0.008, \mathrm{p}=0.039$ and $\mathrm{p}<0.001$, respectively, data not shown). 
Predictive and Prognostic Significance of p27, Akt, PTEN and PI3K Expression in HER2-Positive Metastatic Breast Cancer

There was no significant correlation between p27,Akt, PTEN and PI3K and TTP and OS (Table 2). As seen in Figure 2, there was a trend toward longer median OS in the PTEN positive group compared with PTEN negative group on the Kaplan-Meier curve, but this trend did not reach statistical signficance $(\mathrm{p}=0.058)$.

Treatment response and survival outcomes according to combined expressions of $p 27$, PTEN and Akt

Twenty-five patients were divided into 8 groups according to their PTEN, p27 and Akt expressions (Table 3). There was no correlation between PTEN/p27 groups and ORR. ORR was significantly lower in the patient group that were not expressing neither PTEN nor Akt (PTEN-/Akt-) compared with other groups (20\% vs $80 \%$, $\mathrm{p}=0.023)$. Although there was no correlation between PTEN/p27 and PTEN/Akt groups and median TTP (Figure $3 \mathrm{a}$ and $3 \mathrm{c}$ ), median OS was significantly shorter in the PTEN-/p27- group compared with other PTEN/p27 groups and in the PTEN-/Akt- group compared with other PTEN/Akt groups (26.4 months vs 76.1 months, $\mathrm{p}=0.005$ and 25.6 months vs 52.0 months, $\mathrm{p}=0.007$, respectively) (Figure $3 \mathrm{~b}$ and $3 \mathrm{~d}$ ).

\section{Discussion}

Although trastuzumab is the mainstay of therapy in breast cancer overexpressing HER2, some patients do not respond to trastuzumab. While primary or "de novo" resistance can be in the form of metastatic/recurrent disease developing under adjuvant trastuzumab therapy in a patient with early breast cancer, it can also occur in the form of unresponsiveness to first-line trastuzumab in a patient with metastatic disease. Secondary or "acquired" resistance is defined as unresponsiveness developing later in a patient with metastatic disease initially responsive to trastuzumab (Wilken and Maihle, 2010). Although there are many mechanisms held responsible for the primary and secondary trastuzumab resistance, the most important one is activation PI3K/Akt signal pathway.

PTEN is the most important tumor suppressor gene that limits the activation of PI3K/Akt signal pathway. PTEN dephosphorylates D3 position of PI3P which is a binding site for Akt to the cell membrane by inhibiting PI3K, thus has an antitumoral effect through inhibition of Akt activity. Furthermore, it controls cell cycle by decreasing cycline D and increasing p27 (Jonason et al., 2007). It was first shown by Nagata et al. (2004) that PTEN activation is needed for the antitumoral effect of trastuzumab and that PTEN deficiency causes unresponsiveness to trastuzumab. In patients with PTEN deficiency, P13K and Akt continue to be stimulated independently from HER2 blockage of trastuzumab. Additionally, Akt also inhibits the antitumoral effect of trastuzumab by decreasing p27 levels (Yakes et al., 2002). Preclinical studies have shown that activated Akt (p-Akt) levels are higher and p27 levels are lower in trastuzumab-resistant cells (Yakes et al., 2002; Nahta et al., 2004).

PTEN loss has been reported in nearly $50 \%$ of sporadic breast cancer and 35-86\% of HER2-positive breast cancer (Berns et al., 2007; Gori et al., 2009; Esteva et al., 2010;
Fabi et al., 2010). PTEN mutations and deletions, loss of heterozygosity, PTEN haplodeficiency and epigenetic PTEN downmodulation are mechanisms claimed to lead to functional PTEN loss (Liu et al., 2009). Staining intensity and staining distribution were used as expression criteria in the initial studies assessing PTEN expression (Perren et al., 1999; Depowski et al., 2001). After Nagata et al. (2004) defined IRS, IRS has been used as expression criterion in many studies. However, staining characteristic does not explain the relatively high loss of PTEN in HER2positive breast cancer compared with sporadic breast cancer, because incidence of PTEN loss was found to be similar to the first studies in sporadic breast cancer in the studies carried out using IRS after 2004 (Saal et al., 2005; Berns et al., 2007). All of these results reflect the biologic diversity and heterogenicity of HER2-positive breast cancer. We found loss of PTEN in 19 of 25 (76\%) patients. In a few studies, p27 deficiency has been reported as 35$100 \%$ (Newman et al., 2001; Spataro et al., 2003), p-Akt positivity as 51-80\% (Fabi et al., 2010; Gori et al., 2009) and PI3K positivity as 46-81\% (Fabi et al., 2009; Song et al., 2010) in HER2-positive breast cancer. In our study, p27 deficiency was noted in 40 of 54 patients $(74.1 \%)$ with HER2-positive MBC. Akt and PI3K and expressions were found to be $70.4 \%$ and $96 \%$, respectively.

Consistent with other studies reporting that addition of trastuzumad to the standard therapy improves survival in MBC (Slamon et al., 2001; Marty et al., 2005), median OS and TTP were significantly longer in the patient group responsive to therapy when compared with the non-responders in our study. Nearly all of our patients were PI3K positive, which made it difficult to statistically interpret the correlation between PI3K expression and response status and survival; therefore, PI3K expression was left outside further statistical interpretation. There was no significant correlation between response to trastuzumab and p27 and Akt. Among the total 25 patients, all of the eight patients who did not respond to trastuzumab-based therapy were PTEN negative and all 6 patients with positive PTEN responded to trastuzumab-based therapy. However, when PTEN expression is analysed regarding both negative/positive status and IRS, it had no significant correlation with response to therapy.

Studies have reported conflicting results regarding prediction of trastuzumab response by the elements of PI3K/Akt pathway. It has been reported that PTEN expression and status of PI3K activating mutation are predictive of trastuzumab response only when evaluated together rather than alone (Berns et al., 2007; Esteva et al., 2010; Dave et al., 2011). On the other hand, Fabi et al. (2010) have reported that PTEN, Akt and PI3K expressions are not associated with response to trastuzumab, neither alone nor when evaluated combined as a co-expression. Similarly, Gori et al. (2009) have reported that PTEN and Akt are not predictive regarding response to trastuzumab-based therapy in metastatic setting and Yonemori et al. (2009) have reported the same results in neoadjuvant setting. There are several reasons for the different conclusions in the studies. First, it is possible that prior therapies have affected the expression status of the elements in the present pathway. 
Another important point is evaluating the trastuzumab response. All clinical studies have used RECIST as the evaluation criteria and most patients receive chemotherapy simultaneously with trastuzumab. While in some studies only patients with complete and partial responses are classified as "responsive to trastuzumab-based therapy", in others, stable disease has been evaluated in the same category. "Response" evaluated with RECIST may not be a good criterion for evaluating sensitivity to trastuzumabbased therapies according to the expression of molecules located in the signal pathways, bacause simultaneously used chemotherapy may mask the real effects of the expressions of these molecules in the evaluation of trastuzumab response. Additionally, theoretically, patients who are more sensitive to trastuzumab may be resistant to chemotherapy and in this case they may be considered "unresponsive" trastuzumab-based therapy. The type of chemotherapeutic agent used may also affect response to therapy. HER2-positive breast tumors respond better to anthracycline-containing therapies and taxanes (Di Leo et al., 2002; Hayes et al., 2007). In our study, no correlation was found between response to trastuzumab and the chemotherapeutic agents used. Moreover, other than ORR, the relationship between DCR and p27, PTEN and Akt was also investigated but a significant correlation was not found (unpublished data).

Currently, it is also another controversy whether the molecules located in the PI3K-Akt pathway have a prognostic value in breast cancer. Depowski et al. (2001), Li et al. (2006) and Razis et al. (2011) have reported that PTEN loss and PI3K mutation are poor prognostic factors in breast cancer. On the other hand, Berns et al. (2007) and Pérez-Tenorio et al. (2007) and Perez et al (2013) (A NEW REFERENCE WAS ADDED!) have found no association between PTEN and PI3K and survival. In the study by Esteva et al. (2010) which is the largest study in the literature including 137 patients with HER2-positive MBC, PTEN loss and PI3K mutation were associated with shorter OS evaluated either alone or in combination. Fabi et al. (2010) have reported that when PTEN is evaluated together with PI3K and Akt, PTEN+/PI3K+ and PTEN+/ $\mathrm{Akt}+$ phenotypes are associated with longer PFS. In one of two studies investigating the effect of p27 expression on survival, while no correlation was found with OS and PFS (Engin et al., 2006), low p27 expression predicted shorter disease-free survival when evaluated with PTEN loss in the other study (Tsutsui et al., 2005).

Regarding the relationship between the survival outcomes in our study and PTEN, p27 and Akt expression, a borderline but statistically insignificant relationship was found between PTEN expression and median OS $(\mathrm{p}=0.058)$. Although a positive trend was noted between p27 and Akt expression positivity and OS, the difference did not reach statistical significance. No correlation was found between the three parameters and TTP. Moreover, in the patient group of 25 evaluated for PTEN expression in our study, co-expressions of p27 and Akt and its association with the treatment response and survival were analyzed. Most of the co-expression groups consisted of PTEN-/p27- $(n=17)$ and PTEN-/Akt+ $(n=13)$ patients. ORR was lower and TTP was shorter in the PTEN-/p27- group but it was not statistically significant; on the other hand, OS was statistically significantly shorter compared with other groups $(p=0.005)$. Since trastuzumab exhibits its antitumor effect by increasing PTEN activation and p27 level, it is not surprising that PTEN-/p27- phenotype is associated with trastuzumab resiatance and consequently with poorer response to therapy and prognosis. When co-expression groups of PTEN and Akt were analyzed, ORR was found to be significantly lower in the PTEN-/ Akt- group compared with other groups $(p=0.023)$. TTP was shorter in the PTEN-/Akt- group compared with other groups, but it was not statistically significant $(\mathrm{p}=0.054)$. On the other hand, OS was significantly shorter in the PTEN-/p27- and PTEN-/Akt- groups compared with other phenotypes ( $\mathrm{p}=0.005$ and 0.007 , respectively).

Akt is the major oncogenic determinant molecule in HER2-positive breast cancer. In the study by Fabi et al (2010), PFS was significantly longer in the PTEN+/pAkt+ group. Authors have attributed the their findings to the association of positive PTEN expression with response to trastuzumab and association of Akt positivity with resistance to chemotherapy. The findings related with the PTEN/Akt group in our study may have more than one explanation. First of all, the number of patients in our study group is small. Secondly, there are many factors affecting trastuzumab response and expressions of molecules located in the PI3K/Akt pathway. The third possible explanation is dephosphorylated Akt in our study. Preclinical data show that in HER2-positive breast cancer Akt is frequently found in the phosphorylated that is activated form (p-Akt). The greater p-Akt expression is, the less is dephosphorylated Akt expression which is the other element in the Akt pool. In our study, PTEN-/Aktgroup had the worst survival and PTEN+/Akt- group the best survival rate. In the study by Fabi et al. (2010), the group with the worst prognosis was the PTEN-/p-Akt+ group and the patient group with the best prognosis was the PTEN+/p-Akt+ group. When the negative correlation between Akt and p-Akt is considered, our results seem consistent with those of Fabi et al. (2010).

In conclusion, loss of PTEN and p27 with positive $\mathrm{PI} 3 \mathrm{~K}$ and Akt expression stand out as the common findings seen in HER2-positive MBC in our study. Although PTEN loss seems to be predictive of shorter survival, it has not reached statistical significance probably due to the small number of patients. On the other hand, when PTEN expression is evaluated together with p27 and Akt expression, it can be helpful in identifying the patients who would benefit from trastuzumab therapy. Since HER2positive breast cancer has different biologic characteristics from other breast cancer subtypes, a better understanding of the relationships between signal pathways that play a role in disease progression and resistance to treatment and of the properties of the molecules in these pathways will lead to the development of more successful treatment strategies as well as identifying the predictive and prognostic biomarkers directed at these therapies.

\section{References}

Berns K, Horlings HM, Hennessy BT, et al (2007). A functional genetic approach identifies the PI3K pathway as a major 
Predictive and Prognostic Significance of p27, Akt, PTEN and PI3K Expression in HER2-Positive Metastatic Breast Cancer

determinant of trastuzumab resistance in breast cancer. Cancer Cell, 12, 395-402.

Chen W, Yang H, Tang WR, Feng SJ, Wei YL (2014). Updated meta-analysis on HER2 polymorphisms and risk of breast cancer: evidence from 32 studies. Asian Pac J Cancer Prev, 15, 9643-7.

Dave B, Migliaccio I, Gutierrez MC, et al (2011). Loss of phosphatase and tensin homolog or phosphoinositol-3 kinase activation and response to trastuzumab or lapatinib in human epidermal growth factor receptor 2-overexpressing locally advanced breast cancers. J Clin Oncol, 29, 166-73.

Depowski PL, Rosenthal SI, Ross JS (2001). Loss of expression of the PTEN gene protein product is associated with poor outcome in breast cancer. Mod Pathol, 14, 672-6.

Di Leo A, Gancberg D, Larsimont D, et al (2002). HER-2 amplification and topoisomerase IIalpha gene aberrations as predictive markers in node-positive breast cancer patients randomly treated either with an anthracycline-based therapy or with cyclophosphamide, methotrexate, and 5-fluorouracil. Clin Cancer Res, 8, 1107-16.

Engin H, Baltali E, Güler N, et al (2006). Expression of PTEN, cyclin D1, P27/KIP1 in invasive ductal carcinomas of the breast and correlation with clinicopathological parameters. Bull Cancer, 93, 21-6.

Esteva FJ, Guo H, Zhang S, et al (2010). PTEN, PIK3CA,p-AKT, and p-p70S6K status: association with trastuzumab response and survival in patients with HER2-positive metastatic breast cancer. Am J Pathol, 177, 1647-56.

Fabi A, Metro G, Di Benedetto A, et al (2010). Clinical significance of PTEN and p-Akt co-expression in HER2positive metastatic breast cancer patients treated with trastuzumab-based therapies. Oncology, 78, 141-9.

Fiszman GL, Jasnis MA (2011). Molecular mechanisms of trastuzumab resistance in HER2 overexpressing breast cancer. Int J Breast Cancer, 2011, 352182.

Gori S, Sidoni A, Colozza M, et al (2009). EGFR, pMAPK, pAkt and PTEN status by immunohistochemistry: correlation with clinical outcome in HER2-positive metastatic breast cancer patients treated with trastuzumab. Ann Oncol, 20, 648-54.

Hayes DF, Thor AD, Dressler LG, et al (2007). HER2 and response to paclitaxel in node-positive breast cancer. $N$ Engl J Med, 357, 1496-506.

Jonason JH, Gavrilova N, Wu M, Zhang H, Sun H (2007). Regulation of SCF(SKP2) ubiquitin E3 ligase assembly and p27(KIP1) proteolysis by the PTEN pathway and cyclin D1 . Cell Cycle, 6, 951-61.

Li SY, Rong M, Grieu F, Iacopetta B (2006). PIK3CA mutations in breast cancer are associated with poor outcome. Breast Cancer Res Treat, 96, 91-5.

Liu P, Cheng H, Roberts TM, Zhao JJ (2009). Targeting the phosphoinositide 3-kinase pathway in cancer. Nat Rev Drug Discov, 8, 627-44.

Liu AN, Sun P, Liu JN, et al (2012). Clinicopathologic characteristics and prognostic factors in patients with operable HER-2 overexpressing breast cancer. Asian Pac J Cancer Prev, 13, 1197-201.

Marty M, Cognetti F, Maraninchi D, et al (2005). Randomized phase II trial of the efficacy and safety of trastuzumab combined with docetaxel in patients with human epidermal growth factor receptor 2-positive metastatic breast cancer administered as first-line treatment: the M77001 study group. J Clin Oncol, 23, 4265-74.

Nagata Y, Lan KH, Zhou X, et al (2004). PTEN activation contributes to tumor inhibition by trastuzumab, and loss of PTEN predicts trastuzumab resistance in patients. Cancer Cell, 6, 117-27.

Nahta R, Takahashi T, Ueno NT, Hung MC, Esteva FJ (2004). $\mathrm{P} 27$ (kip1) down-regulation is associated with trastuzumab resistance in breast cancer cells. Cancer Res, 64, 3981-6.

Nahta R, Esteva FJ (2006). HER2 therapy: molecular mechanisms of trastuzumab resistance. Breast Cancer Res, 8, 215.

Newman L, Xia W, Yang HY, et al (2001). Correlation of p27 protein expression with HER-2/neu expression in breast cancer. Mol Carcinog, 30, 169-75.

Perez EA, Dueck AC, McCullough AE, et al (2103). Impact of PTEN protein expression on benefit from adjuvant trastuzumab in early-stage human epidermal growth factor receptor 2-positive breast cancer in the North Central Cancer Treatment Group N9831 trial. J Clin Oncol, 31, 2115-22.

Pérez-Tenorio G, Alkhori L, Olsson B, et al (2007). PIK3CA mutations and PTEN loss correlate with similar prognostic factors and are not mutually exclusive in breast cancer. Clin Cancer Res, 13, 3577-84.

Perren A, Weng LP, Boag AH, et al (1999). Immunohistochemical evidence of loss of PTEN expression in primary ductal adenocarcinomas of the breast. Am J Pathol, 155, 1253-60.

Prat A, Baselga J (2013). Dual human epidermal growth factor receptor 2 (HER2) blockade and hormonal therapy for the treatment of primary HER2-positive breast cancer: one more step toward chemotherapy-free therapy. J Clin Oncol, 31, 1703-6.

Razis E, Bobos M, Kotoula V, et al (2011). Evaluation of the association of PI3KCA mutations and PTEN loss with efficacy of trastuzumab therapy in metastatic breast cancer. Breast Cancer Res Treat, 128, 447-56.

Ross JS, Slodkowska EA, Symmans WF, et al (2009). The HER-2 receptor and breast cancer: ten years of targeted anti-HER-2 therapy and personalized medicine. Oncologist, 14, 320-68.

Saal LH, Holm K, Maurer M, et al (2005). PIK3CA mutations correlate with hormone receptors, node metastasis, and ERBB2, and are mutually exclusive with PTEN loss in human breast carcinoma. Cancer Res, 65, 2554-9.

Slamon DJ, Leyland-Jones B, Shak S, et al (2001). Use of chemotherapy plus a monoclonal antibody against HER2 for metastatic breast cancer that overexpresses HER2. $N$ Engl J Med, 344, 783-92.

Song CH, Park SY, Eom KY, et al (2010). Potential prognostic value of heat-shock protein 90 in the presence of phosphatidylinositol-3-kinase overexpression or loss of PTEN, in invasive breast cancers. Breast Cancer Res, 12, 20.

Spataro VJ, Litman H, Viale G, et al (2003). Decreased immunoreactivity for $\mathrm{p} 27$ protein in patients with earlystage breast carcinoma is correlated with HER-2/neu overexpression and with benefit from one course of perioperative chemotherapy in patients with negative lymph node status: results from International Breast Cancer Study Group Trial V. Cancer, 97, 1591-600.

Spector NL, Blackwell KL (2009). Understanding the mechanisms behind trastuzumab therapy for human epidermal growth factor receptor 2-positive breast cancer. $J$ Clin Oncol, 27, 5838-47.

Tsutsui S, Inoue H, Yasuda K, et al (2005). Inactivation of PTEN is associated with a low $\mathrm{p} 27 \mathrm{Kip} 1$ protein expression in breast carcinoma. Cancer, 104, 2048-53.

Wilken JA, Maihle NJ (2010). Primary trastuzumab resistance: new tricks for an old drug. Ann Y D Acad Sci, 1210, 53-65.

Yakes FM, Chinratanalab W, Ritter CA, et al (2002). Herceptininduced inhibition of phosphatidylinositol-3 kinase and Akt Is required for antibody-mediated effects on $\mathrm{p} 27$, cyclin D1, and antitumor action. Cancer Res, 62, 4132-41.

YonemoriK,Tsuta K,Shimizu C,etal (2009).Immunohistochemical expression of PTEN and phosphorylated Akt are not correlated with clinical outcome in breast cancer patients treated with trastuzumab-containing neo-adjuvant chemotherapy. Med Oncol, 26, 344-9. 